\title{
The polynomially bounded perfect matching problem is in $\mathrm{NC}^{2 \star}$
}

\author{
Manindra Agrawal ${ }^{1}$, Thanh Minh Hoang ${ }^{2}$, and Thomas Thierauf ${ }^{3}$ \\ 1 IIT Kanpur, India \\ 2 Ulm University, Germany \\ 3 Aalen University, Germany
}

\begin{abstract}
The perfect matching problem is known to be in $\boldsymbol{\Upsilon}$, in randomized NC, and it is hard for NL. Whether the perfect matching problem is in $\mathbf{N C}$ is one of the most prominent open questions in complexity theory regarding parallel computations.

Grigoriev and Karpinski [GK87] studied the perfect matching problem for bipartite graphs with polynomially bounded permanent. They showed that for such bipartite graphs the problem of deciding the existence of a perfect matchings is in $\mathbf{N C}^{2}$, and counting and enumerating all perfect matchings is in $\mathbf{N C}^{3}$. For general graphs with a polynomially bounded number of perfect matchings, they show both problems to be in $\mathbf{N C}^{3}$. In this paper we extend and improve these results. We show that for any graph that has a polynomially bounded number of perfect matchings, we can construct all perfect matchings in $\mathbf{N C}^{2}$. We extend the result to weighted graphs.
\end{abstract}

\section{Introduction}

Whether there is an NC-algorithm for testing if a given graph contains a perfect matching is an outstanding open question in complexity theory. The problem of deciding the existence of a perfect matching in a graph is known to be in 9 [Edm65], in randomized $\mathbf{N C}^{2}$ [MVV87], and in nonuniform SPL [ARZ99]. This problem is very fundamental for other computational problems (see e.g. [KR98]). Another reason why a derandomization of the perfect matching problem would be very interesting is, that it is a special case of the polynomial identity testing problem.

Since no NC-algorithm is known for testing the existence of perfect matchings in a common graph, some special cases of the perfect matching problem have been investigated intensively. For example, NCalgorithms have been found the perfect matching problem for regular bipartite graphs [LPV81], dense graphs [DHK93], strongly chordal graphs [DK86] and planar graphs [Kas67,Vaz89]. The unique perfect matching problem is considered in [HMT06].

Grigoriev and Karpinski [GK87] considered the perfect matching problem for bipartite graphs with polynomially bounded number of perfect matchings, i.e. a

\footnotetext{
* Supported by DFG grant Scho 302/7-1.
} 
promise problem. They showed that the decision version of the perfect matching problem for such graphs is solvable in $\mathbf{N C}^{2}$. and that all perfect matchings for such graphs can be constructed in $\mathbf{N C}^{3}$. For general graphs, their techniques bring both problems into $\mathbf{N C}^{3}$.

We extend the result of Grigoriev and Karpinski [GK87] to arbitrary weighted graphs and improve the upper bound to $\mathbf{N C}^{2}$. That is, we show that on input of some graph $G$ one can construct all perfect matchings of $G$ in $\mathbf{N C}^{2}$, if $G$ has a polynomially bounded number of perfect matchings. We show the result for bipartite graphs in Section 3 and then extend it to general graphs in Section 4. In Section 5 we generalize our techniques to graphs with polynomially bounded weights.

When we restrict ourselves to the decision version or the counting version of the problem, we get logspace counting classes inside $\mathbf{N C}^{2}$ as upper bounds for these problems.

\section{Preliminaries}

Let $G=(V, E)$ be an undirected graph. A matching in $G$ is a set $M \subseteq E$, such that no two edges in $M$ have a vertex in common. A matching $M$ is called perfect if every vertex occurs as an endpoint of some edge in $M$. Define

$$
P M(G)=\{M \mid M \text { is a perfect matching in } G\} .
$$

Bipartite Graphs. Let $G$ be bipartite, that is we can partition the nodes into $V=L \cup R$ such that there are no edges in $L$ and in $R$. We assume w.l.o.g. that $|L|=\mid R=n$, otherwise $G$ has no perfect matching. The bipartite adjacency matrix of $G$ is the $n \times n$ matrix $A=\left(a_{i, j}\right)$, where

$$
a_{i, j}= \begin{cases}1 & \text { if }(i, j) \in E, \text { for } i \in L \text { and } j \in R, \\ 0 & \text { otherwise. }\end{cases}
$$

The bipartite Tutte matrix of $G$ is the $n \times n$ matrix $T=\left(t_{i, j}\right)$, where

$$
t_{i, j}=a_{i, j} x_{i, j}
$$

for indeterminates $x_{i, j}$. The determinant of $T$ is

$$
\operatorname{det}(T)=\sum_{\pi \in S_{n}} \operatorname{sign}(\pi) \prod_{i=1}^{n} a_{i, \pi(i)} x_{i, \pi(i)} .
$$

$\operatorname{det}(T)$ is a multi-linear polynomial. Each non-vanishing term $\operatorname{sign}(\pi) \prod_{i=1}^{n} x_{i, \pi(i)}$ corresponds to one perfect matching $M_{\pi}=\{(i, \pi(i)) \mid 1 \leq i \leq n\} \in P M(G)$. In particular we have

Theorem 1 (Tutte 1952). Let $G$ be a bipartite graph. $G$ has a perfect matching iff $\operatorname{det}(T) \neq \mathbf{0}$. 
General Graphs. Let $G$ be a graph with $n$ nodes. W.l.o.g. assume that $n$ is even, otherwise $G$ has no perfect matchings. Let $A=\left(a_{i, j}\right)$ be the $n \times n$ adjacency matrix of $G$. Note that $A$ is symmetric. The skew-symmetric Tutte matrix of $G$ is the $n \times n$ matrix $T=\left(t_{i, j}\right)$, where

$$
t_{i, j}= \begin{cases}a_{i, j} x_{i, j}, & \text { if } i \leq j, \\ -a_{j, i} x_{j, i}, & \text { otherwise }\end{cases}
$$

for indeterminates $x_{i, j}$. The Pfaffian of $T$ is

$$
\operatorname{pf}(T)=\sum_{M \in P M(G)} \operatorname{sign}(M) \cdot \prod_{\substack{(i, j) \in M \\ i<j}} a_{i, j} x_{i, j}
$$

The sign is defined as follows. Consider perfect matching

$$
M=\left\{\left(i_{1}, j_{1}\right),\left(i_{2}, j_{2}\right), \ldots,\left(i_{k}, j_{k}\right)\right\} \in P M(G)
$$

for $k=n / 2$. By convention, we have $i_{l}<j_{l}$ for all $l$. The sign of $M$ is defined as the sign of the permutation

$$
\left(\begin{array}{ccccccc}
1 & 2 & 3 & 4 & \cdots & n-1 & n \\
i_{1} & j_{1} & i_{2} & j_{2} & \cdots & i_{k} & j_{k}
\end{array}\right) \in S_{n}
$$

It is known that the sign of $M$ does not depend on the order in which the edges are given, i.e. the sign is well defined.

$\operatorname{pf}(T)$ is a multi-linear polynomial. Each non-vanishing term $\operatorname{sign}(M)$.

$\prod x_{i, j}$ corresponds to one perfect matching $M \in P M(G)$. The Pfaffian $\underset{\substack{(i, j) \in M \\ i<j}}{ }$

and the determinant of a matrix are known to be closely related.

Theorem 2. $\operatorname{det}(T)=\mathrm{pf}^{2}(T)$.

In particular we have

Theorem 3 (Tutte 1952). Graph $G$ has a perfect matching iff $\operatorname{det}(T) \neq \mathbf{0}$.

Linear Algebra. The following matrix is called a Vandermonde matrix

$$
V=\left(\begin{array}{cccc}
1 & 1 & \cdots & 1 \\
a_{1} & a_{2} & \cdots & a_{n} \\
a_{1}^{2} & a_{2}^{2} & \cdots & a_{n}^{2} \\
\vdots & \vdots & & \vdots \\
a_{1}^{n-1} & a_{2}^{n-1} & \cdots & a_{n}^{n-1}
\end{array}\right)
$$

It is known that

$$
\operatorname{det}(V)=\prod_{i \neq j}\left(a_{i}-a_{j}\right)
$$


Hence, in the case when $a_{1}, a_{2}, \ldots, a_{n}$ are pairwise distinct the matrix $V$ is nonsingular. The inverse can be written as

$$
V^{-1}=\frac{1}{\operatorname{det}(V)} \operatorname{adj}(V),
$$

where $\operatorname{adj}(V)$ is the adjoint of $V$.

Complexity Classes. The classes $\mathbf{N} \mathbf{C}^{k}$, for fixed $k$, consists of families of Boolean circuit with $\wedge^{-}, \vee$-gates of fan-in 2 , and $\neg$-gates, of depth $O\left(\log ^{k} n\right)$ and of polynomial size. $\mathbf{N C}=\cup_{k \geq 0} \mathbf{N C}^{k}$.

Standard arithmetic operations like addition, subtraction, multiplication and integer division are known to be in $\mathbf{N C}^{1}$. Many problems from linear algebra like computing powers of a matrix are in $\mathbf{N C}^{2}$. A break-through result was that the determinant of a matrix is computable in $\mathbf{N C}^{2}$ [Ber84].

For a nondeterministic Turing machine $M$, we denote the number of accepting and rejecting computation paths on input $x$ by $\operatorname{acc}_{M}(x)$ and by $r e j_{M}(x)$, respectively. The difference of these two quantities is $g a p_{M}$, i.e., for all $x: \operatorname{gap}_{M}(x)=$ $\operatorname{acc}_{M}(x)-r e j_{M}(x)$. The complexity class GapL is defined as the set of all functions $\operatorname{gap}_{M}(x)$, where $M$ is a nondeterministic logspace bounded Turing machine. Most notably, we have

Theorem 4. [Dam91,Tod91,Vin91,Val92] The determinant of an integer matrix is complete for GapL.

And similarly for the Pfaffian we have

Theorem 5. [MSV99] The Pfaffian of an integer matrix is complete for $\mathbf{G a p L}$

GapL is closed under addition, subtraction, and multiplication. It is not known to be closed under integer division. In particular, consider the inverse of matrix like in the above example, $V^{-1}=\frac{1}{\operatorname{det}(V)} \operatorname{adj}(V)$. The entries of the adjoint matrix are determinants and can therefore be computed in GapL. But we don't know whether the entries of $V^{-1}$ can be computed in GapL too because of the division by $\operatorname{det}(V)$. However, with the adjoint matrix we have the entries of $\operatorname{det}(V) V^{-1}$ in GapL.

The class $\mathbf{C}_{=} \mathbf{L}$ (Exact Counting in Logspace) is the class of sets $A$ for which there exists a function $f \in \mathbf{G a p L}$ such that $\forall x: x \in A \Longleftrightarrow f(x)=0$. A problem complete for $\mathbf{C}_{=} \mathbf{L}$ is the singularity problem, where one has to decide whether the determinant of an integer matrix is zero. $\mathbf{C}_{=} \mathbf{L}$ is closed under union and intersection, but is not known to be closed under complement.

Problems that can be expressed as a (unbounded) boolean combination of sets from $\mathbf{C}_{=} \mathbf{L}$ are captured by the class $\mathbf{A} \mathbf{C}^{0}\left(\mathbf{C}_{=} \mathbf{L}\right)$ of sets being $\mathbf{A} \mathbf{C}^{0}$-reducible to $\mathbf{C}_{=} \mathbf{L}$. Allender, Beals, and Ogihara [ABO99] defined and studied this class. They show for example that the problem to decide whether a system of linear equations has a solution is complete for $\operatorname{AC}^{0}\left(\mathbf{C}_{=} \mathbf{L}\right)$. We have the following inclusions.

$$
\mathbf{N L} \subseteq \mathbf{C}_{=} \mathbf{L} \subseteq \mathbf{A C}^{0}\left(\mathbf{C}_{=} \mathbf{L}\right) \subseteq \mathbf{N C}^{2}
$$


Cook [Coo85] defined the class DET as the class of sets that are $\mathbf{N C}^{1}$ reducible to the determinant. Since the determinant is complete for GapL, we denote DET by $\mathbf{N C}^{1}(\mathbf{G a p L})$. We have $\mathbf{N C} \mathbf{C}^{1}(\mathbf{G a p L}) \subseteq \mathbf{N} \mathbf{C}^{2}$.

\section{Bipartite Graphs}

In this section we prove the following theorem.

Theorem 6. All perfect matchings of a bipartite graph with a polynomially bounded number of perfect matchings can be constructed in $\mathbf{N C}^{2}$.

Let $G=(V, E)$ be a bipartite graph with $|V|=2 n$ nodes and let $A=\left(a_{i, j}\right)$ be the bipartite adjacency matrix of $G$. Let $p$ be a polynomial and assume that $G$ has at most $p(n)$ perfect matchings. Define

$$
b_{i, j}^{(m)}(x)=a_{i, j} p_{i, j} x^{m^{n i+j} \bmod r},
$$

where $p_{i, j}$ are pairwise different primes, $x$ is an indeterminate, $r$ is a prime such that $r>n^{2} p^{2}(n)$, and $0 \leq m<r$. We can choose $\max \left\{p_{i, j} \mid 1 \leq i, j \leq n\right\}=$ $O\left(n^{3}\right)$ by the Prime Number Theorem. For $1 \leq m<r$ define matrices

$$
B_{m}(x)=\left(b_{i, j}^{(m)}(x)\right) .
$$

The determinant of $B_{m}(x)$ is a polynomial $d_{m}(x)$, where

$$
\begin{aligned}
d_{m}(x)=\operatorname{det}\left(B_{m}(x)\right) & =\sum_{\pi \in S_{n}} \operatorname{sign}(\pi) \prod_{i=1}^{n} a_{i, \pi(i)} p_{i, \pi(i)} x^{m^{n i+\pi(i)} \bmod r} \\
& =\sum_{\pi \in S_{n}} \operatorname{sign}(\pi)\left(\prod_{i=1}^{n} a_{i, \pi(i)} p_{i, \pi(i)}\right) x^{e_{m}(\pi)}
\end{aligned}
$$

where $e_{m}(\pi)=\sum_{i=1}^{n}\left(m^{n i+\pi(i)} \bmod r\right)$ are the exponents of $x$ in $d_{m}(x)$.

The crucial point here is, that the summands of $e_{m}(\pi)$ are taken modulo $r$. Therefore the degree of polynomial $d_{m}(x)$ is bounded by $D=n(r-1)$, which is a polynomial in $n$. Without the $\bmod r$ we would have exponential degree. On the other hand, without the $\bmod r$, for any $\pi \in S_{n}$ the exponent of $x$ is unique. We show in the following that this also holds modulo $r$, at least for some $m$.

Lemma 1. Let $\pi_{1}, \ldots, \pi_{t} \in S_{n}$ for some $t \leq p(n)$. Then there exists an $m<r$ such that $e_{m}\left(\pi_{i}\right) \neq e_{m}\left(\pi_{j}\right)$, for all $i \neq j$.

Proof. The values $e_{m}\left(\pi_{i}\right)$ can be seen as evaluations of polynomials over the field $\mathbb{Z}_{r}$ in the following way. Define

$$
q_{\pi}(z)=\sum_{i=1}^{n} z^{n i+\pi(i)} .
$$


Then we have $e_{m}\left(\pi_{i}\right) \equiv q_{\pi}(m)(\bmod r)$, for any $m$. To prove the lemma, we have to show that $q_{\pi_{i}}(m) \not \equiv q_{\pi_{j}}(m)(\bmod r)$, for some $m<r$ and for all $i \neq j$.

Notice first that $q_{\pi_{i}} \neq q_{\pi_{j}}$, for any $i \neq j$. Now the degree of the $q$-polynomials is bounded by $n^{2}+n \leq 2 n^{2}$. Hence any two of them can agree on at most $2 n^{2}$ points. Thus in any field of size larger than $\left(\begin{array}{l}t \\ 2\end{array}\right) 2 n^{2}$ we have a point where all polynomials $q_{\pi_{i}}$ pairwise differ. Note that

$$
\left(\begin{array}{l}
t \\
2
\end{array}\right) 2 n^{2} \leq t^{2} n^{2} \leq p^{2}(n) n^{2}<r .
$$

Hence there is an appropriate $m$ in $\mathbb{Z}_{r}$.

It follows that if $G$ has $t$ perfect matchings for some $t \leq p(n)$, then there exists an $m<r$ such that polynomial $d_{m}(x)$ has precisely $t$ terms. That is,

$$
d_{m}(x)=\sum_{k=0}^{D} c_{k}^{(m)} x^{k}
$$

where precisely $t$ of the coefficients $c_{k}^{(m)}$ are non-zero. Moreover, the non-zero coefficients are of the form

$$
c_{k}^{(m)}=\operatorname{sign}(\pi) \prod_{i=1}^{n} p_{i, \pi(i)}
$$

for some $\pi \in S_{n}$ such that $k=e_{m}(\pi)$. We want to compute these coefficients.

Define the Vandermonde matrix $V=\left(v_{i, j}\right)$ by $v_{i, j}=i^{j}$, for $0 \leq i, j \leq D$. Define vectors

$$
\begin{aligned}
\boldsymbol{d}_{m} & =\left(\begin{array}{llll}
d_{m}(0) & d_{m}(1) & \cdots & d_{m}(D)
\end{array}\right)^{T} \\
\boldsymbol{c}_{m} & =\left(\begin{array}{llll}
c_{0}^{(m)} & c_{1}^{(m)} & \cdots & c_{D}^{(m)}
\end{array}\right)^{T}
\end{aligned}
$$

The evaluation of polynomial $d_{m}(x)$ at points $0, \ldots, D$ can now be written as

$$
\boldsymbol{d}_{m}=V \boldsymbol{c}_{m}
$$

Therefore we obtain the coefficient vector by the equation

$$
\boldsymbol{c}_{m}=V^{-1} \boldsymbol{d}_{m}
$$

By the latter equation, $\boldsymbol{c}_{m}$ can be computed in $\mathbf{N C}^{2}$.

Lemma 2. $c_{m} \in \mathrm{NC}^{2}$.

Proof. The matrices $V$ and $B_{m}(x)$ can be computed in $\mathbf{N C}^{1}$ for any $x \leq D$. Vector $\boldsymbol{d}_{m}$ can be computed by computing the determinant of matrix $B_{m}(x)$ for different values of $x$, which is in $\mathbf{N C}^{2}$ by Theorem 4 . Also, $V^{-1}$ can be computed in $\mathrm{NC}^{2}$. 
The final step is to determine the prime factors $p_{i, j}$ of the non-zero coefficients in $\boldsymbol{c}_{m}$, because these factors define perfect matchings as explained above. Given a non-zero $c_{k}^{(m)}$, we can test in $\mathbf{N C ^ { 1 }}$ whether $c_{k}^{(m)} \equiv 0\left(\bmod p_{i, j}\right)$ since all $p_{i, j}$ are $O\left(n^{3}\right)$. In summary, we can construct all perfect matchings of $G$ in $\mathbf{N C}^{2}$ if we have the right value of $m$.

To find the right value for $m$, we compute $\boldsymbol{c}_{m}$ for all $m \in\{1, \ldots, r-1\}$ in parallel. We can take any $m$ such that $\boldsymbol{c}_{m}$ has a maximum number of non-zero entries. The procedure remains in $\mathbf{N C}^{2}$.

In fact, we get a slightly better upper bound. Note first that the entries of all vectors $\operatorname{det}(V) \boldsymbol{c}_{m}=\operatorname{adj}(V) \boldsymbol{d}_{m}$ can be computed in GapL. Having all these values, the remaining computation can be done in $\mathbf{N C}^{1}$. Recall in particular that integer division is in $\mathbf{N C}^{1}$ [CDL01].

Suppose we want to know only whether there exists some perfect matching (decision problem) or count the number of perfect matchings (counting problem). For the decision problem it suffices to determine whether $\boldsymbol{c}_{m}$ is non-zero for some $m$. Note that this is equivalent to $\operatorname{det}(V) \boldsymbol{c}_{m}$ being non-zero. For the counting problem we have to count the number of non-zero entries of $\boldsymbol{c}_{m}$, for an $m$ such that $\boldsymbol{c}_{m}$ has a maximum number of non-zero entries.

Corollary 1. For bipartite graphs with a polynomially bounded number of perfect matchings

1. the decision problem is in $\mathbf{c o} \mathbf{C}_{=} \mathbf{L}$,

2. the counting problem is in $\mathbf{A C}^{0}\left(\mathbf{C}_{=} \mathbf{L}\right)$,

3. the construction problem is in $\mathbf{N C}^{1}(\mathbf{G a p L})$.

\section{General Graphs}

In this section we extend Theorem 6 to non-bipartite graphs.

Theorem 7. All perfect matchings of a graph with a polynomially bounded number of perfect matchings can be constructed in $\mathbf{N C}^{2}$.

Let $G=(V, E)$ be an undirected graph with $|V|=n$ nodes. We assume that $n$ is even, otherwise $G$ has no perfect matchings. Let $A=\left(a_{i, j}\right)$ be the adjacency matrix of $G$. Let $p$ be a polynomial and assume that $G$ has at most $p(n)$ perfect matchings. We define matrices $B_{m}(x)=\left(b_{i, j}^{(m)}(x)\right)$ in a similar fashion as before. The definition is now according to the Tutte matrix of $G$ :

$$
b_{i, j}^{(m)}(x)= \begin{cases}a_{i, j} p_{i, j} x^{m^{n+j} \bmod r}, & \text { if } i \leq j \\ -a_{j, i} p_{j, i} x^{m^{n j+i} \bmod r}, & \text { otherwise }\end{cases}
$$

for pairwise different primes $p_{i, j}$ of size $O\left(n^{3}\right)$, an indeterminate $x$, a prime $r$ such that $r>n^{2} p^{2}(n)$, and $1 \leq m<r$. 
The Pfaffian of $B_{m}(x)$ is a polynomial $p_{m}(x)$, where

$$
\begin{aligned}
p_{m}(x)=\operatorname{pf}\left(B_{m}(x)\right) & =\sum_{M \in P M(G)} \operatorname{sign}(M) \cdot \prod_{\substack{(i, j) \in M \\
i<j}} a_{i, j} p_{i, j} x^{m^{n i+j} \bmod r} \\
& =\sum_{M \in P M(G)} \operatorname{sign}(M) \cdot\left(\prod_{\substack{(i, j) \in M \\
i<j}} a_{i, j} p_{i, j}\right) x^{e_{m}(M)},
\end{aligned}
$$

where

$$
e_{m}(M)=\sum_{\substack{(i, j) \in M \\ i<j}}\left(m^{n i+j} \bmod r\right)
$$

are the exponents of $x$ in $p_{m}(x)$. Similar as in Lemma 1 we have that there is some $m<r$ where the exponents $e_{m}(M)$ pairwise differ.

Note that $e_{m}(M) \leq(r-1) n / 2$. Let $D=(r-1) n / 2$. Then we can write

$$
p_{m}(x)=\sum_{k=0}^{D} c_{k}^{(m)} x^{k}
$$

Define the Vandermonde matrix $V=\left(v_{i, j}\right)$ by $v_{i, j}=i^{j}$, for $0 \leq i, j \leq D$. Define vectors

$$
\begin{aligned}
\boldsymbol{p}_{m} & =\left(\begin{array}{llll}
p_{m}(0) & p_{m}(1) & \cdots & p_{m}(D)
\end{array}\right)^{T} \\
\boldsymbol{c}_{m} & =\left(\begin{array}{llll}
c_{0}^{(m)} & c_{1}^{(m)} & \cdots & c_{D}^{(m)}
\end{array}\right)^{T}
\end{aligned}
$$

As in the bipartite case we have $\boldsymbol{p}_{m}=V \boldsymbol{c}_{m}$, from which we get $\boldsymbol{c}_{m}=V^{-1} \boldsymbol{p}_{m}$. By Theorem $5, \boldsymbol{c}_{m}$ can be computed in $\mathbf{N C}^{2}$.

Corollary 2. For graphs with a polynomially bounded number of perfect matchings,

1. the decision problem is in $\mathbf{c o} \mathbf{C}_{=} \mathbf{L}$,

2. the counting problem is in $\mathbf{A C}^{0}\left(\mathbf{C}_{=} \mathbf{L}\right)$,

3. the construction problem are in $\mathbf{N C}^{1}(\mathbf{G a p L})$.

\section{$5 \quad$ Weighted Graphs}

In this section we extend Theorem 7 to graphs with small weights. Let $G=(V, E)$ be an undirected graph with $|V|=n$ nodes. Let $A=\left(a_{i, j}\right)$ be the adjacency matrix of $G$ and $W=\left(w_{i, j}\right)$ be the symmetric matrix that gives weight $w_{i, j}$ to edge $(i, j)$, where all weights are polynomially bounded in $n$.

There are several variants of problems we might consider: the minimal perfect matching problem asks for a perfect matching of minimum weight. In its promise version, we assume that there are at most polynomially many perfect matching of minimum weight. Analogously, there is the maximum perfect matching problem. 
But actually, we can solve a more general problem. It suffices that for some weight $w$ there are at most $p(n)$ many perfect matching of weight $w$, for some polynomial $p$.

Theorem 8. Let $G$ be a weighted graph with polynomially bounded weights such that $G$ has a polynomially bounded number of perfect matchings of some weight $w$. Then all perfect matchings of $G$ of weight $w$ can be constructed in $\mathbf{N C}^{2}$.

Define matrices $B_{m}(x, y)=\left(b_{i, j}^{(m)}(x, y)\right)$ in two variables $x$ and $y$ that incorporate the weights of $G$ :

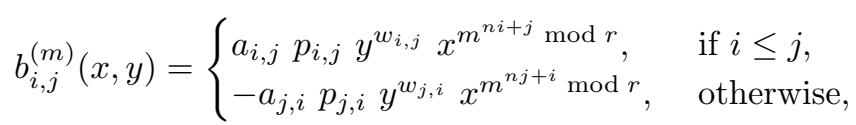

for pairwise different primes $p_{i, j}$ of size $O\left(n^{3}\right)$, indeterminates $x$ and $y$, a prime $r$ such that $r>n^{2} p^{2}(n)$, and $1 \leq m<r$.

The Pfaffian of $B_{m}(x, y)$ is a polynomial $p_{m}(x, y)$, where

$$
\begin{aligned}
p_{m}(x, y)=\operatorname{pf}\left(B_{m}(x, y)\right) & =\sum_{M \in P M(G)} \operatorname{sign}(M) \cdot \prod_{\substack{(i, j) \in M \\
i<j}} a_{i, j} p_{i, j} y^{w_{i, j}} x^{m^{n i+j} \bmod r} \\
& =\sum_{M \in P M(G)} \operatorname{sign}(M) \cdot\left(\prod_{\substack{(i, j) \in M \\
i<j}} a_{i, j} p_{i, j}\right) y^{w(M)} x^{e_{m}(M)}
\end{aligned}
$$

where $e_{m}(M)=\sum_{\substack{(i, j) \in i_{i<j} \\ i<}}\left(m^{n i+j} \bmod r\right)$. By a similar argument as in Lemma 1 we have that there is some $m<r$ where the exponents $e_{m}(M)$ pairwise differ, and this suffices for our purpose.

The degree of $x$ in $p_{m}(x, y)$ is bounded by $(r-1) n / 2$. Let $d=(r-1) n / 2+1$, so that the degree of $x$ in $p_{m}(x, y)$ is strictly less than $d$. We transform $p_{m}(x, y)$ into polynomial $P_{m}(x)$ with just one variable by setting

$$
P_{m}(x)=p_{m}\left(x, x^{d}\right)
$$

Then we have

$$
P_{m}(x)=\sum_{M \in P M(G)} \operatorname{sign}(M) \cdot\left(\prod_{\substack{(i, j) \in M \\ i<j}} a_{i, j} p_{i, j}\right) x^{d w(M)+e_{m}(M)}
$$

By our choice of $d$ we have $d>e_{m}(M)$. Let $w$ be any fixed weight and consider a perfect matching $M$ of weight $w$. Then we have

$$
d w<d w+e_{m}(M)<d(w+1) .
$$

That is, the degrees of $x$ in $P_{m}(x)$ for perfect matchings of different weights $w$ are in disjoint intervals of the form $(d w, d(w+1))$. Let $D$ be the degree of $P_{m}(x)$. 
We have $D \leq d w_{\max }$, where $w_{\max }$ is the maximum weight of any matching. Note that $w_{\max } \leq \max \left\{w_{i, j} \mid 1 \leq i, j \leq n\right\} n / 2$. Let

$$
P_{m}(x)=\sum_{k=0}^{D} c_{k}^{(m)} x^{k} .
$$

We have seen in Section 4 how to determine the coefficients $c_{k}^{(m)}$ and how to get the perfect matchings from these coefficients in $\mathbf{N C}^{2}$. Note that the perfect matchings of weight $w$ are represented by the coefficients $c_{k}^{(m)}$ for $d w<k<$ $d(w+1)$.

Now, if there are at most $p(n)$ perfect matchings of weight $w$, then all of these will be listed by our $\mathbf{N C}^{2}$-circuit. Note however that we might list perfect matchings of other weights as well. In case that the promise is for the minimum (or maximum) weight perfect matching, we may discard non-optimal perfect matchings.

\section{Open Problems}

We have the polynomial bound on the number of perfect matchings given as a promise. Clearly the ultimate goal is to get rid of the promise and to put the perfect matching problem in $\mathbf{N C}^{2}$. We conjecture that, modulo some small modifications, our approach works for the general case. It remains to prove this.

\section{Acknowledgments}

We thank Eric Allender for clarifying various subtleties concerning logspace computations.

\section{References}

[ABO99] E. Allender, R. Beals, and M. Ogihara. The complexity of matrix rank and feasible systems of linear equations. Computational Complexity, 8:99 -126, 1999.

[ARZ99] E. Allender, K. Reinhardt, and S. Zhou. Isolating, matching, and counting: uniform and nonuniform upper bounds. Journal of Computer and System Sciences, 59:164-181, 1999.

[Ber84] S. Berkowitz. On computing the determinant in small parallel time using a small number of processors. Information Processing Letters, 18:147-150, 1984.

[CDL01] A. Chiu, G. Davida, and B. Litow. Division in logspace-uniform NC ${ }^{1}$. RAIRO Theoretical Informatics and Applications, 35:259-276, 2001.

[Coo85] S. Cook. A taxonomy of problems with fast parallel algorithms. Information and Control, 64:2-22, 1985.

[Dam91] C. Damm. DET $=\mathrm{L}^{(\# L)}$. Technical Report Informatik-Preprint 8, Fachbereich Informatik der Humboldt-Universität zu Berlin, 1991. 
[DHK93] E. Dahlhaus, P. Hajnal, and M. Karpinski. On the parallel complexity of hamiltonian cycles and matching problem in dense graphs. Journal of Algorithms, 15:367-384, 1993.

[DK86] E. Dahlhaus and M. Karpinski. The matching problem for strongly chordal graphs is in NC. Technical Report 855-CS, University of Bonn, 1986.

[Edm65] J. Edmonds. Maximum matching and a polyhedron with 0-1 vertices. Journal of Research National Bureau of Standards, 69:125-130, 1965.

[GK87] D. Grigoriev and M. Karpinski. The matching problem for bipartite graphs with polynomially bounded permanent is in NC. In 28th Annual IEEE Symposium on Foundations of Computer Science (FOCS), pages 166-172. IEEE Computer Society Press, 1987.

[HMT06] T. M. Hoang, M. Mahajan, and T. Thierauf. On the Bipartite Unique Perfect Matching Problem. In Automata, Languages and Programming, 33rd International Colloquium, (ICALP), Lecture Notes in Computer Science 4051, pages 453-464. Springer-Verlag, 2006.

[Kas67] P. W. Kastelyn. Graph theory and crystal physics. In F. Harary, editor, Graph Theory and Theoretical Physics, pages 43-110. Academic Press, 1967.

[KR98] M. Karpinski and W. Rytter. Fast Parallel Algorithms for Graph Matching Problems. Oxford University Press, 1998.

[LPV81] G. Lev, M. Pippenger, and L. Valiant. A fast parallel algorithm for routing in permutation networks. IEEE Transactions on Computers, C-30:93-100, 1981.

[MSV99] M. Mahajan, P. Subramanya, and V Vinay. A combinatorial algorithm for pfaffians. In 5th Annual International Conference on Computing and Combinatorics (COCOON), Lecture Notes in Computer Science 1627, pages 134143. Springer-Verlag, 1999.

[MVV87] K. Mulmuley, U. Vazirani, and V. Vazirani. Matching is as easy as matrix inversion. In 19th ACM Symposium on Theory of Computing, pages 345-354. ACM Press, 1987.

[Tod91] S. Toda. Counting problems computationally equivalent to the determinant. Technical Report CSIM 91-07, Dept. of Computer Science and Information Mathematics, University of Electro-Communications, Chofu-shi, Tokyo 182, Japan, 1991.

[Val92] L. Valiant. Why is boolean complexity theory difficult. In M.S. Paterson, editor, Boolean Function Complexity, London Mathematical Society Lecture Notes Series 169. Cambridge University Press, 1992.

[Vaz89] V. Vazirani. NC algorithms for computing the number of perfect matchings in $K_{3,3}$-free graphs and related problems. Information and computation, 80(2):152-164, 1989.

[Vin91] V Vinay. Counting auxiliary pushdown automata and semi-unbounded arithmetic circuits. In 6th IEEE Conference on Structure in Complexity Theory, pages 270-284, 1991. 